\title{
I \\ ON A TROPICAL VERSION OF THE JACOBIAN CONJECTURE
}

\author{
DIMA GRIGORIEV AND DANYLO RADCHENKO
}

\begin{abstract}
We prove for a tropical rational map that if for any point the convex hull of Jacobian matrices at smooth points in a neighborhood of the point does not contain singular matrices then the map is an isomorphism. We also show that a tropical polynomial map on the plane is an isomorphism if all the Jacobians have the same sign (positive or negative). In addition, for a tropical rational map we prove that if the Jacobians have the same sign and if its preimage is a singleton at least at one regular point then the map is an isomorphism.
\end{abstract}

\section{INTRODUCTION}

We provide two criteria for a tropical map of $n$-dimensional real spaces to be an isomorphism. The basic concepts of tropical (or min-plus) mathematics one can find in [6]. The first criterion concerns tropical rational maps and states that if at any point the convex hull of Jacobian matrices at smooth points in a neighborhood of the point does not contain singular matrices then the map is an isomorphism (Proposition 10 and Theorem 21). Note that there is totally a finite number of Jacobian matrices corresponding to polyhedra on which the tropical rational map is linear. We also show that this criterion is not necessary (even for 2-dimensional tropical polynomial isomorphisms).

The second criterion deals with tropical polynomial maps and holds for 2dimensional real spaces. It claims that if the Jacobians at all the smooth points have the same sign (either positive or negative) then the tropical polynomial map is an isomorphism (Theorem 5). We construct an example which demonstrates that this criterion fails for tropical rational 2-dimensional maps as well as for tropical polynomial 3-dimensional ones. In addition, for a tropical rational map we prove that if the Jacobians at all the smooth points have the same sign and if its preimage is a singleton at least at one regular point then the map is an isomorphism (Theorem 4).

\section{WEAK VERSION OF THE TROPICAL JACOBIAN CONJECTURE}

By a tropical algebraic rational function (in $n$ variables) we mean a function from $\mathbb{R}^{n}$ to $\mathbb{R}$ of the form $\min \left\{A_{1}, \ldots, A_{k}\right\}-\min \left\{B_{1}, \ldots, B_{l}\right\}$ where $A_{1}, \ldots, A_{k}$, $B_{1}, \ldots, B_{l}$ are linear functions with rational coefficients (when the coefficients at the variables are positive integers one talks about tropical rational functions). They play the role of a tropical analog of algebraic functions (cf. 3]). In fact, one could stick with more generally, real coefficients.

From a different point of view, defining a tropical algebraic rational function amounts to giving a finite decomposition $\mathbb{R}^{n}=\bigcup_{i} C_{i}$, where each $C_{i}$ is a closed $n$-dimensional polyhedron and the interiors of $C_{i}$ and $C_{j}$ do not intersect if $i \neq j$,

2010 Mathematics Subject Classification 14T05 
and specifying a linear function $f_{i}$ on each $C_{i}$ in such a way that $f_{i}$ and $f_{j}$ agree on $C_{i} \cap C_{j}$. Similar description applies in general to tropical algebraic rational mappings from $\mathbb{R}^{n}$ to $\mathbb{R}^{m}$. We will call $\left(f_{i}, C_{i}\right)$ or just $f_{i}$ the linear pieces of $f$.

Observe that if a tropical algebraic rational map $\mathbb{R}^{n} \rightarrow \mathbb{R}^{n}$ is a bijection then its inverse is also a tropical algebraic rational map. In this case we call such a map an isomorphism.

Proposition 1. If a tropical algebraic rational map $f: \mathbb{R}^{n} \rightarrow \mathbb{R}^{n}$ is locally injective, then it is an isomorphism.

Proof. Let $f_{1}, \ldots, f_{N}$ be the linear pieces of $f$. Since $f$ is locally injective, each $f_{i}$ has to be invertible, and hence for any compact $K \subset \mathbb{R}^{n}$ we have that $f_{i}^{-1}(K)$ is compact. Therefore, $f^{-1}(K) \subseteq \bigcup_{i=1}^{N} f_{i}^{-1}(K)$ is compact, and thus $f$ is a proper mapping. By the domain invariance theorem [1] the mapping $f$ is locally a homeomorphism. The result then follows directly from [5, Th. 2] that, specialized to our setting, says that if a continuous mapping $f: \mathbb{R}^{n} \rightarrow \mathbb{R}^{n}$ is a local homeomorphism and is proper, then it is a global homeomorphism.

This result can be treated as a weak tropical version of the Jacobian conjecture in the sense that for tropical rational algebraic maps local invertibility implies global invertibility. The problem remains how to tell if a given map is locally an isomorphism. There is a simple sufficient condition for local invertibility of Lipschitz mappings that can be easily stated in our case (it is not hard to show that tropical rational algebraic maps are Lipschitz). Let $f: \mathbb{R}^{n} \rightarrow \mathbb{R}^{n}$ be a tropical algebraic rational mapping with linear pieces $\left(f_{i}, C_{i}\right), i=1 \ldots, N$. For any point $x \in \mathbb{R}^{n}$ let $I=\left\{i \mid x \in C_{i}\right\}$. We then define $\partial_{f}(x)$ to be the convex hull of the set of differentials (Jacobian matrices) of $f_{i}, i \in I$ (in the space of matrices $\operatorname{Mat}_{n \times n}(\mathbb{R})$ ). The following is a special case of the inverse function theorem for Lipschitz mappings proved by Clarke [2, Th. 1].

Theorem 2. If $f: \mathbb{R}^{n} \rightarrow \mathbb{R}^{n}$ is a tropical algebraic rational mapping and the set $\partial_{f}\left(x_{0}\right)$ contains only nonsingular matrices, then $f$ is a homeomorphism in a neighborhood of $x_{0}$.

Note, however, that the sufficient condition in Theorem 2 for $f$ to be an isomorphism is not necessary, as the following example shows.

Example 3. We construct a tropical polynomial isomorphism $f: \mathbb{R}^{2} \rightarrow \mathbb{R}^{2}$ as a composition of two tropical polynomial maps (isomorphisms): a lower-triangular map

$$
(x, y) \mapsto(x, y+\min \{\alpha x, \beta x\}), \alpha<\beta
$$

and an upper-triangular one:

$$
(x, y) \mapsto(x+\min \{a y, b y\}, y), a<b .
$$

Then $f(x, y)=$

$$
\begin{array}{lll}
f_{1}:=(x+a(y+\alpha x), y+\alpha x) & \text { if } \quad x>0, y+\alpha x>0 ; \\
f_{2}:=(x+b(y+\alpha x), y+\alpha x) & \text { if } \quad x>0, y+\alpha x<0 ; \\
f_{3}:=(x+a(y+\beta x), y+\beta x) & \text { if } \quad x<0, y+\beta x>0 ; \\
f_{4}:=(x+b(y+\beta x), y+\beta x) & \text { if } \quad x<0, y+\beta x<0 .
\end{array}
$$


The corresponding matrices of differentials at the origin are as follows:

$$
\left(\begin{array}{cc}
1+a \alpha & a \\
\alpha & 1
\end{array}\right),\left(\begin{array}{cc}
1+b \alpha & b \\
\alpha & 1
\end{array}\right),\left(\begin{array}{cc}
1+a \beta & a \\
\beta & 1
\end{array}\right),\left(\begin{array}{cc}
1+b \beta & b \\
\beta & 1
\end{array}\right) .
$$

The sum of the second and the third matrices is singular when $(\beta-\alpha)(b-a)=4$ (in particular, one can put $\beta=b=2, \alpha=a=0)$. Thus, $\partial_{f}(0,0)$ contains a singular matrix.

\section{Strong VERSION OF THE TROPICAL JACOBIAN CONJECTURE}

Denote by $J_{i}, 1 \leq i \leq N$ the determinants (Jacobians) of the differentials of $f_{i}$. If $f$ is an isomorphism then all $J_{i}$ must have the same sign (either positive or negative). This follows from a general result about homeomorphisms (see, for example, [4, Th. 5.22]) or can be verified directly by comparing the Jacobians $J_{i}, J_{j}$ for a pair of $f_{i}, f_{j}$ defined on adjacent ( $n$-dimensional) polyhedra separated by a common $(n-1)$-dimensional facet. Observe that the condition on $\partial_{f}(p)$ in Theorem 2 implies that all the Jacobians have the same sign since for a pair of real matrices $A, B$ with $\operatorname{det}(A B)<0$ there exists $0<t<1$ such that $\operatorname{det}(t A+(1-$ t) $B)=0$.

The condition that all $J_{i}$ are nonzero and have the same sign is not sufficient to guarantee that $f$ is an isomorphism (see Example 6 below), but we will now show that it is almost sufficient. In analogy to the case of smooth maps, we call $y \in \mathbb{R}^{n}$ a regular value of $f$, if it does not belong to the set $\bigcup_{i=1}^{N} f\left(\partial C_{i}\right)$. A version of Sard's theorem is true (and is trivial) in this case: the set of regular values is dense in $\mathbb{R}^{n}$.

Theorem 4. A necessary and sufficient condition for a tropical algebraic rational mapping $f: \mathbb{R}^{n} \rightarrow \mathbb{R}^{n}$ to be an isomorphism is that all Jacobians $J_{i}$ have the same sign, and that $\left|f^{-1}\left(y_{0}\right)\right|=1$ for at least one regular value $y_{0}$.

Proof. The necessity is clear. To prove sufficiency we will use standard techniques from topological degree theory (for a general reference, see [7, Ch. IV]).

Without loss of generality we can assume that all Jacobians are positive. Since $f$ is proper, it has a well-defined degree $\operatorname{deg}(f)$ (as a mapping from the one-point compactification of $\mathbb{R}^{n}$ to itself), and by computing it using the preimage of the regular value $y_{0}$ we get $\operatorname{deg}(f)=1$. This, in turn, implies that $\left|f^{-1}(y)\right|=1$ holds for any regular value $y$. The proof would then be complete if we could show that $f$ is an open mapping: indeed, if $f\left(x_{1}\right)=f\left(x_{2}\right)$ for some $x_{1} \neq x_{2}$, then $\left|f^{-1}(y)\right| \geq 2$ for all $y$ in a sufficiently small neighborhood of $f\left(x_{1}\right)$, which would contradict the above computation for regular $y$.

To show that $f$ is open we use an argument similar to the one used in [8, Proof of Thm. 3]. Note that $\left|f^{-1}(y)\right| \leq N$ for all $y$, where $N$ is the number of linear pieces of $f$. Therefore, for any $x \in \mathbb{R}^{n}$ we can find a small neighborhood $U$ of $x$ such that $f^{-1}(f(x)) \cap U=\{x\}$. Let $V$ be a small open ball around $f(x)$ that does not intersect $f(\partial U)$. Then for some smooth point $x_{0} \in U \cap f^{-1}(V)$ we have that $f\left(x_{0}\right)$ is a regular value, and hence $\operatorname{deg}\left(f, U, f\left(x_{0}\right)\right)=1$. Since $\operatorname{deg}(f, U, p)$ only depends on the connected component of $\mathbb{R}^{n} \backslash f(\partial U)$ to which $p$ belongs, we get that $\operatorname{deg}(f, U, y)=1$ for all $y \in V$. But if $\operatorname{deg}(f, U, y) \neq 0$, then $y \in f(U)$ (see [7. Cor. IV.2.5(3)]), so that $V \subset f(U)$. Thus $f$ is an open mapping.

Let us remark that this result gives a simple computational approach to checking that a given tropical rational algebraic map $f$ is an isomorphism. Indeed, to check 
the above conditions one only needs to compute the set of linear functions $f_{i}$ and the preimage of a generic point $y_{0}$, but both of these tasks are easy to do using linear programming.

It turns out that if $n=2$ and $f$ is a tropical algebraic mapping, so that each coordinate of $f$ is concave and piecewise linear, then a stronger result is true.

Theorem 5. If $f: \mathbb{R}^{2} \rightarrow \mathbb{R}^{2}$ is a tropical algebraic mapping (i.e., $f=\left(\phi_{1}, \phi_{2}\right)$ where $\phi_{i}$ are tropical algebraic functions) such that all the Jacobians $J_{i}$ have the same sign, then $f$ is an isomorphism.

Proof. For $a \in \mathbb{R}$ such that the preimage $\phi_{1}^{-1}(a) \subset \mathbb{R}^{2}$ is non-empty, it is the boundary of the convex polygon $P:=\left\{x \mid \phi_{1}(x) \geq a\right\}$. Consider the supporting to $P$ lines $L_{1}, \ldots, L_{m}$. Either $m=2$ and $L_{1}, L_{2}$ are parallel or one can renumber $L_{1}, \ldots, L_{m}$ to make their slopes in the clock-wise order (with respect to an arbitrary point of $P$ ).

In the latter case $\phi_{1}^{-1}(a)$ is a connected polygonal line with the consecutive edges (some of them, perhaps, unbounded) lying on $L_{1}, \ldots, L_{m}$, respectively. Due to the condition imposed on the Jacobians $J_{i}$ in Theorem $5, \phi_{2}$ is strictly monotone along $\phi_{1}^{-1}(a)$. Therefore, $\phi_{1}^{-1}(a)$ has to be unbounded with just two unbounded edges $L_{1}, L_{m}$, and $\phi_{2}$ restricted to $\phi_{1}^{-1}(a)$ (being a piecewise linear function with a finite number of pieces) provides an isomorphism with $\mathbb{R}$. Thus, we conclude that $f$ is an isomorphism if $\phi_{1}^{-1}(a)$ is connected (or empty) for all $a \in \mathbb{R}$ by Proposition 1 . By symmetry, $f$ is also an isomorphism if $\phi_{2}^{-1}(a)$ is connected (or empty) for all $a \in \mathbb{R}$.

Now we study the case when $m=2$ and $L_{1}, L_{2}$ are parallel. Let $\phi_{i}=$ : $\min \left\{A_{i, 1}, \ldots, A_{i, s_{i}}\right\}, 1 \leq i \leq 2$. Then each linear function $A_{1, q}, 1 \leq q \leq s_{1}$ is constant on $L_{1}$. Hence the graph $\Phi_{1} \subset \mathbb{R}^{3}$ of $\phi_{1}$ is the Cartesian product of $L_{1}$ and a convex polygonal line $Q \subset \mathbb{R}^{2}$ which can be treated as the graph of a univariate concave piecewise linear function. The latter function is bounded from above since $\Phi_{1}$ contains $L_{1}, L_{2}$. Therefore, $\phi_{1}$ attains its maximum on a line $L$ parallel to $L_{1}, L_{2}$.

Thus, it remains to consider the case when for both $\phi_{1}, \phi_{2}$ there exist $a_{1}, a_{2} \in \mathbb{R}$ such that $\phi_{1}^{-1}\left(a_{1}\right), \phi_{2}^{-1}\left(a_{2}\right)$ are disconnected, so they consist of pairs of parallel lines. So, $L$ separates two adjacent pieces on which $\phi_{1}$ is linear with their differentials being collinear with a negative coefficient of collinearity. If at some point $y$ of $L$ the piecewise linear function $\phi_{2}$ is non-singular (thereby, is linear in a neighborhood of $y$ ) then (the only) two linear maps $f_{i}, f_{j}$ defining $f$ in a neighborhood of $y$ have the Jacobians $J_{i}, J_{j}$ with opposite signs contrary to the condition of Theorem 5 .

Otherwise, if $\phi_{2}$ has an edge on $L$ separating two pieces on which $\phi_{2}$ is linear, then for two linear maps $f_{i}, f_{j}$ which define $f$ in a neighborhood of any point of this edge their Jacobians $J_{i}, J_{j}$ both vanish. Obviously, $\phi_{1}$ can't attain its maximum on a 2-dimensional facet since the differential of $\phi_{1}$ would vanish on it. We get a contradiction with the supposition that there exist $a_{1}, a_{2} \in \mathbb{R}$ such that $\phi_{1}^{-1}\left(a_{1}\right), \phi_{2}^{-1}\left(a_{2}\right)$ are disconnected.

The simple condition of Theorem [5] is no longer sufficient for $n \geq 3$, as the following example shows.

Example 6. We will construct a tropical polynomial mapping $f: \mathbb{R}^{n} \rightarrow \mathbb{R}^{n}, n \geq 3$, for which the Jacobians $J_{i}>0$ for all $i$, but which is not an isomorphism. 
Clearly, it is enough to construct such an example for $n=3$. We start with a two-dimensional tropical rational mapping $g: \mathbb{R}^{2} \rightarrow \mathbb{R}^{2}$ defined by

$$
(x, y) \quad \mapsto \quad(|x|-|y|,|x+y|-|x-y|) .
$$

Note that the Jacobian of each linear piece of $g$ is equal to 2 , but $g(x, y)=$ $g(-x,-y)$, so that $g$ is not injective. To construct $f$ we start with the mapping that sends $(x, y, z)$ to $(g(x, y), z)$ and then apply elementary piecewise linear transformations to get rid of all the subtractions in the min-plus form. More precisely, let us define $h: \mathbb{R}^{3} \rightarrow \mathbb{R}^{3}$ by

$$
(x, y, z) \mapsto(-|y|-|x+y|+z,-|x|-|x-y|+z,-|x+y|-|x|+z) .
$$

One can check that $h$ is a tropical Laurent polynomial map (since $-|x|=\min (-x, x)$ is), its Jacobian is equal to 2 at every smooth point, and $h(x, y, z)=h(-x,-y, z)$. To get the mapping $f$, we compose $h$ with $z \mapsto z+2 x+2 y$ to obtain

$$
\begin{aligned}
f(x, y, z)= & (\min (2 y, 0)+\min (2 x+2 y, 0)+z+x, \\
& \min (2 x, 0)+\min (2 x, 2 y)+z+y, \\
& \min (2 x, 0)+\min (2 x+2 y, 0)+z+y) .
\end{aligned}
$$

Then $f$ is a tropical polynomial map whose Jacobian at every smooth point is equal to 2 , but it is not an isomorphism since $f(-x,-y, z+4 x+4 y)=f(x, y, z)$.

Acknowledgements. The first author is grateful to ANR/DFG grant SYMBIONT. The second author is grateful to Max Planck Institute for Mathematics in Bonn for its hospitality and financial support.

\section{REFERENCES}

[1] L. E. J. Brouwer, Zur Invarianz des n-dimensionalen Gebiets, Mathematische Annalen 72, (1912) 55-56.

[2] F. H. Clarke, On the inverse function theorem, Pacific Journal of Mathematics, 64, 1 (1976) 97-102.

[3] D. Grigoriev, Tropical Newton-Puiseux polynomials, Lect. Notes Comput. Sci., 11077 (2018) 177-186.

[4] S. Hencl, P. Koskela, Lectures on mappings of finite distortion, Lecture Notes in Mathematics, 2096. Springer, Cham, 2014.

[5] C.-W. Ho, A note on Proper Maps, Proc. AMS, 51, 1 (1975) 237-241.

[6] D. Maclagan, B. Sturmfels, Introduction to Tropical Geometry, American Math. Society, 2015.

[7] E. Outerelo, J. M. Ruiz, Mapping Degree Theory, American Math. Society, 2009.

[8] D. Radchenko, Approximation by maps with nonnegative Jacobian, Math. Notes, 93, 1-2 (2013) 297-307.

CNRS, Mathématiques, Université de Lille, Villeneuve D’AscQ, 59655, France

E-mail address: dmitry.grigoryev@univ-lille.fr

Max Planck Institute for Mathematics, Bonn, Germany

E-mail address: danradchenko@gmail.com 